\title{
Do we always need computed tomography to detect and treat parapneumonic pleural effusion?
}

\section{To the Editors:}

We read with interest the article by MoFFETT et al. [1] that was recently published in the European Respiratory Journal. In this retrospective analysis, conducted using two databases of 1,460 pneumonia patients enrolled from one single centre, the investigators studied the parapneumonic pleural effusion (PPE) linear cut-off by chest computed tomography (CCT) that indicates the need for thoracentesis. The authors concluded that patients with community-acquired pneumonia (CAP) with a PPE measuring $<2.5 \mathrm{~cm}$ by CCT can be managed without the need for thoracentesis [1].

We agree with the statement of the authors affirming the importance of rapid detection and execution of thoracentesis in PPE [1]; however we would like to point out some concerns about the potential impact of this study in clinical practice.

CCT represents one of the most important advances in modern radiology [2] and is generally considered the gold standard for studying the lungs and pleura. However, as reminded by BRENNER et al. [2], compared with plain-film radiography, CCT involves much higher doses of radiation (with a ratio of 1:100 to $1: 1,000)$, resulting in a marked increase in radiation exposure in patients, even when using the new low-dose CCT technique. Data from epidemiological studies suggest that the estimated risks associated with low radiation exposure are not hypothetical, since the organ doses correlated to a common CCT study result in an increased risk of cancer [2]. Moreover, compared to other forms of imaging, such as ultrasonography, CCT is a high time and resource consuming examination. For these reasons, considering the risk-benefit assessment, a CCT scan should only be ordered for selected patients in whom there is clear medical evidence that they are in need of this examination (e.g. unexplained hypoxia, pulmonary embolism, empyema or cancer). As reported by HoOPER et al. [3], the add-on value of a CCT scan is related to the evaluation of complex pleuroparenchymal disease as it is better at delineating loculated pleural collection, parenchymal consolidation and the mediastinum. However, loculated effusions are a major limitation of the dimensional criteria proposed by MOFFET et al. [1] for assessing the need for thoracentesis. Despite such effusions being excluded from the analysis, this issue is of major clinical interest. In fact, loculated effusions, even those of small dimensions, should always undergo pleural procedures for the elevated risk of empyema.

Thoracic ultrasonography is an imaging tool that is gaining importance in clinical practice. Ultrasound can be performed at the bedside without radiation, and ultrasound guidance is strongly recommended for all pleural procedures for pleural effusion [3]. The important role of ultrasound in the early investigation of pleural effusion is clearly stated in the recent
British Thoracic Society guidelines [3] and specifically recommended for the management of PPE by LIGHT [4]. Moreover, thoracic ultrasonography differentiates between pleural effusion and pleural thickening, and detects septations within pleural fluid with a greater sensitivity than CCT scanning, thus being effective in the early stratification of empyema $[3,5]$.

It is noteworthy that in their retrospective database analysis MOFFET et al. [1] hypothesised "that [...] CCT [...] would provide an attractive early alternative in patients who have already received a $\mathrm{CCT}^{\prime \prime}$, and the authors included patients that underwent CCT for a discretional decision of emergency room and hospital provider's; we presume for reasons often unrelated to the study of pleural effusion. The conclusion of this study may erroneously lead to the clinical message that millions of patients suffering from CAP every year should undergo CCT to detect and manage PPE.

We conclude that rapid detection and evacuation of PPE is a major issue in the management of CAP. However, after the execution of chest radiographs, ultrasonography should be the first-line exam to detect and manage PPE, leaving CCT to selected patients.

\section{G.F. Sferrazza Papa, F. Di Marco, P. Carlucci, L. Colombo, M. Mondoni and S. Centanni \\ Clinica di Malattie dell'Apparato Respiratorio, Ospedale San Paolo, Università degli Studi di Milano, Milan, Italy.}

Correspondence: G.F. Sferrazza Papa, Clinica di Malattie dell'Apparato Respiratorio, Ospedale San Paolo, Università degli Studi di Milano, Milan, Italy. E-mail: franz83@ymail.com

Statement of Interest: None declared.

\section{REFERENCES}

1 Moffett BK, Panchabhai TS, Anaya E, et al. Computed tomography measurements of parapneumonic effusion indicative of thoracentesis. Eur Respir J 2011; 38: 1406-1411.

2 Brenner DJ, Hall EJ. Computed tomography - an increasing source of radiation exposure. $N$ Engl J Med 2007; 357: 2277-2284.

3 Hooper C, Lee YC, Maskell N, et al. Investigation of a unilateral pleural effusion in adults: British Thoracic Society Pleural Disease Guideline 2010. Thorax 2010; 65: Suppl. 2, ii4-ii17.

4 Light RW. Parapneumonic effusions and empyema. Proc Am Thorac Soc 2006; 3: 75-80.

5 Brutsche MH, Tassi GF, Györik S, et al. Treatment of sonographically stratified multiloculated thoracic empyema by medical thoracoscopy. Chest 2005; 128: 3303-3309.

DOI: 10.1183/09031936.00224011 